\title{
Dinámicas de relación sociedad-Estado en la educación en Veracruz, México
}

\author{
Felipe Hevia*
}

Perfiles Latinoamericanos

Flacso México

\begin{abstract}
Resumen
El interés de este artículo es analizar el tema de la participación en México. Por medio del estudio de caso de la educación, se busca caracterizar las dinámicas de la relación sociedad-Estado más allá de los dispositivos institucionales. La metodología es mixta: se realizaron grupos focales y entrevistas, se aplicó un cuestionario a consejos escolares $(\mathrm{N}=127)$, y se construyeron bases de datos sobre peticiones a las instancias oficiales $(\mathrm{N}=2783)$ y notas de prensa $(\mathrm{N}=1326)$. Los resultados describen tres dinámicas de relación sociedad-Estado: 1) participación reglamentada que se expresa en el desempeńo de los Consejos Escolares de Participación Social, 2) relaciones particularistas que se basan en la búsqueda de una relación directa ciudadano-autoridad por medio de peticiones y solicitudes, y 3) relaciones contenciosas-disruptivas caracterizadas por acciones de protesta. Se discuten los efectos de estas dinámicas en la reproducción de las asimetrías de poder y en la construcción de capacidades de respuesta diferenciada por parte del gobierno, y se propone una agenda de investigación.
\end{abstract}

\begin{abstract}
There is interest in analyzing participation in Mexico. Through the case study of education, the objective is to analyze different patterns of relationship between society and State beyond institutional devices. The methodology is mixed: we applied focus groups and interviews, a questionnaire to school boards $(\mathrm{N}=127)$ was applied, databases petitions to the authorities $(\mathrm{N}=2783)$ and newspaper notes $(\mathrm{N}=1326)$ were built. The results describe three dynamics of relationship between society and State: 1) regulated participation, which is expressed in the performance of the School Councils of Social Participation, 2) particularistic relations, which are based on finding a direct relationship between citizen-authority through petitions and applications, and 3) contentious-disruptive relationships characterized by protest actions. The effects of these dynamics are discussed, in terms of reproducing the asymmetries of power and building differentiated response capabilities by the government, and a research agenda is proposed.
\end{abstract}

Palabras clave: participación social, participación de los padres, gestión educacional, política educacional, relación padres-escuela, administración de la educación, relaciones sociedad-Estado. Keywords: Social participation, parent participation, educational management, educational policy, parent-school relationship, educational administration, State-society relationship.

* Doctor en antropología, Centro de Investigaciones y Estudios Superiores en Antropología Social (CIESAS). Profesor-investigador de CIESAS-Golfo. 


\section{Introducción ${ }^{1}$}

E

las discusiones recientes sobre los procesos de profundización democrática y las relaciones sociedad-Estado en América Latina, sobresale la creciente importancia de la participación de los ciudadanos en diversas instancias de decisión además del voto. Ese tipo de participación se puede definir de manera amplia como el derecho de grupos y personas a incidir en el espacio público (Hevia \& Vergara-Lope, 2011: p. 10) y se expresa en una variada gama de instituciones y prácticas que buscan que los ciudadanos puedan representar sus intereses e influir en la toma de decisiones, así como monitorear y evaluar políticas y programas en elecciones (participación política o electoral) y entre elecciones (participación ciudadana). La inclusión de la participación en el interés académico es visible en una variada literatura que incluye los análisis sobre calidad e innovación democrática (Isunza \& Gurza, 2010; Parés, 2009), capacidades estatales y gobernanza (Aguilar, 2011; Alonso, 2007), y construcción del espacio público (Cunill, 2012; Merino, 2010).

La relevancia del tema en la academia es en parte reflejo del giro participativo que ha caracterizado parte de los procesos de construcción democrática en América Latina. Este proceso ha sido discontinuo y accidentado, y se ha caracterizado por una disputa entre diversos proyectos políticos que usan conceptos como participación o ciudadanía pero con significados muy diversos, generándose así una serie de confluencias perversas en relación con estos conceptos (Dagnino, 2006).

El giro participativo incluyó la creación de canales institucionalizados de participación en la mayoría de las constituciones de los países de la región, así como la generación de legislación secundaria orientada normar instrumentos de democracia directa y otros dispositivos de participación (Hevia, 2010; Welp \& Serduilt, 2009). Sin embargo, su implementación y uso han sido escasos, e incluso muchas de estas innovaciones han implicado en la práctica procesos de centralización del poder en los ejecutivos (Altman, 2005; Lissidini, Welp \& Zovatto, 2008). De igual manera, al mismo tiempo que dos países pioneros en la inclusión de instancias participativas en sus marcos legales, como Colombia y Brasil, estaban discutiendo nuevas leyes y lineamientos buscando fortalecer los dispositivos de

1 Este documento forma parte del proyecto "Sistemas de atención ciudadana en el campo educativo en Veracruz" financiado por el CIESAS entre 2009 y 2012, cuyos resultados ampliados se presentan en Hevia (2014). Quiero agradecer a Frida García, Antonia Lara y Ernesto García por el trabajo en la elaboración de las bases de datos contenidas en este documento, así como a los dictaminadores anónimos del artículo, cuyas agudas observaciones mejoraron sustantivamente la versión final. 
participación institucional (PNUD, 2015), en sus calles se debatían problemas de letigimidad política de las instituciones democráticas.

En la bibliografía que analiza el giro participativo y las innovaciones democráticas sobresale el análisis de dispositivos como los presupuestos participativos (Avritzer, 2010), el referéndum, l iniciativa popular y otros instrumentos de democracia directa (Altman, 2005; Lissidini et al., 2008), consejos gestores a nivel territorial o sectorial (Zaremberg, 2012), o consejos consultivos (Hevia, Vergara-Lope \& Ávila, 2011). A esta literatura se vienen sumando con mayor frecuencia estudios más bien críticos que pretenden incorporar estos procesos participativos en dinámicas mayores, como sus vínculos con los procesos de representación e intermediación política (Gurza \& Zaremberg, 2015; Selee \& Peruzzotti, 2009), así como analizar los vínculos que estas experiencias tienen con otras prácticas políticas, tal como las estructuras y movimientos populares y sus ligas con prácticas clientelares (Auyero, Lapegna \& Poma, 2009). Estos análisis comparten un fuerte componente de análisis empírico y el interés por examinar los procesos participativos desde una visión más amplia de relación sociedad-Estado, disminuyendo así el riesgo de establecer interpretaciones que generen expectativas desproporcionadas sobre los efectos de la participación en la democracia o las políticas redistributivas (Font, 2015).

Como veremos a continuación, este artículo busca sumarse a esta corriente ampliando la mirada hacia otras formas de relación sociedad-Estado, además de las generadas por los dispositivos de participación institucional, lo que permita valorar la capacidad de tales dispositivos para cumplir con las expectativas que generan. Para ello utilizamos el caso de la educación.

En los debates educativos, la importancia de la participación no ha estado ausente. Tal como la evidencia disponible sugiere, en los procesos de aprendizaje confluyen factores escolares y extraescolares (Backhoff et al., 2007), y en ambos la participación juega un papel relevante. Dentro de los factores escolares uno de los elementos que influye en el logro educativo es la gestión o gobernanza escolar (Gann, 1998). Diversos estudios muestran que una gestión colaborativa tiene un efecto positivo en el incremento de los aprendizajes en las escuelas tanto en los países desarrollados (Paletta, 2012) como en aquellos en vías de desarrollo (López, 2010; Rojas \& Ramírez, 2007). Uno de los factores centrales en la gobernanza escolar es la participación social y el involucramiento de los padres de familia. Por ello en una buena cantidad de países el andamiaje legal de la gobernanza escolar ha sufrido importantes cambios legales (Bush \& Glover, 2013; Castanheira \& Costa, 2011) y es una discusión que ha generado fuertes controversias (Gleeson \& Husbands, 2003).

Dentro de los factores extraescolares, la literatura sobre participación social en educación se ha enfocado en cuatro grandes temáticas: en primer lugar, los 
efectos del involucramiento parental en los procesos de aprendizajes (Carvalho, 2000; Epstein, 2001; Hornby \& Lafaele, 2011). En segundo, la relación entre participación e incremento del logro educativo (Delgado-Gaitán, 1991; Driessen, Smit \& Sleegers, 2005), incluyendo análisis para el caso mexicano (Huerta, 2010). En tercero, las relaciones entre escuela, padres y comunidad (Bauch \& Goldring, 1998; Dietz, 2004), y finalmente el análisis de los dispositivos de participación en los procesos de gestión escolar, como los consejos y comités escolares (Anderson, 2005; Henderson \& Mapp, 2002; Martínez, Bracho \& Martínez, 2010). Estas temáticas coinciden en el peso de la participación social para fomentar los aprendizajes, mejorar la gestión, construir ciudadanía y garantizar así los derechos a la educación y a la participación, derechos fundamentales protegidos por diversos tratados internacionales, y por los cuerpos legales de cada país.

En México, las autoridades educativas han realizado esfuerzos en fechas recientes por proteger este derecho por medio de la expedición de lineamientos para los Consejos Escolares de Participación Social en las escuelas (sep, 2010) y constituye un campo de preocupación regular de la investigación educativa. La mayoría de trabajos se han centrado en los dispositivos de participación, especialmente los Consejos de Participación Social, en diferentes dimensiones (Observatorio Ciudadano de la Educación, 2008): su descripción y andamiaje institucional (Canales, 2006; Latapí, 1997), su inserción en políticas específicas como el Programa Escuelas de Calidad (Santizo, 2006), sus capacidades para exigir cuentas (Martínez et al., 2010), el desempeńo de estos Consejos en escuelas (Santizo, 2011; Zurita, 2013), así como en algunos municipios (Estrada, 2008). Muchos de estos análisis coinciden en la limitación de estos instrumentos para generar incidencia (Estrada, 2014: p. 737).

Por otro lado, existe investigación enfocada al estudio de otras dimensiones de la participación social más allá de los dispositivos institucionales. Aquí se incluye el análisis de las prácticas cotidianas en la escuela (Rockwell, 1995); proyectos pedagógicos alternativos desarrollados por la Coordinadora Nacional de Trabajadores de la Educación (CNTE) en Oaxaca, Michoacán y Chiapas; experiencias de organizaciones sociales en Puebla, Querétaro, Zacatecas y el Distrito Federal, y escuelas zapatistas en Chiapas (Alaníz, 2013). De igual manera, otros ejemplos de prácticas participativas en educación se encuentra en la importante corriente de educación popular (Martin, 2010: p. 47).

Este artículo pretender aportar a estas discusiones puesto que tiene por objetivo describir las dinámicas de relación que existen entre los ciudadanos y las autoridades educativas, buscando examinar, además de la participación social vía consejos, otros modelos recurrentes de interacción en la educación pública en México. En este sentido, el argumento central es el siguiente: es posible iden- 
tificar al menos tres dinámicas de relación entre ciudadanos y gobernantes en el campo educativo, los que denominamos dinámicas de participación reglamentada, particularistas y contenciosas-disruptivas. Estas relaciones se combinan de múltiples maneras en la vida cotidiana y están representadas, respectivamente, en el uso de las instancias institucionales de consulta y participación existentes en el campo educativo, en acciones de peticiones y solicitudes directas a la autoridad política, y en acciones disruptivas como protestas y manifestaciones. Esto nos permite discutir los efectos de estas relaciones para la gestión educativa en México, en particular a los retos que se encuentran en la implementación de las reformas constitucionales a la educación de 2013.

De igual manera, en las conclusiones se pretende discutir la pertinencia de analizar la participación institucional como una dinámica más, entre otras, de relación sociedad-Estado, buscando ampliar así los vínculos de la participación institucional con otros procesos políticos.

\section{Método}

Los resultados aquí presentados responden a una investigación que se llevó a cabo entre 2009 y 2012 en el estado de Veracruz, y que utilizó diversas estrategias de recolección y análisis de información, privilegiando una matriz analítica centrada en la antropología política y una estrategia mixta de investigación (Hevia, 2014). Esto porque en su interior se viene desarrollando una importante discusión sobre los procesos de formación y construcción cotidiana del Estado (Sharma \& Gupta, 2006). Esta perspectiva privilegia una perspectiva relacional para caracterizar relaciones sociedad-gobierno en múltiples escalas (Hevia, 2009) y se concentra en los procesos de construcción del Estado por medio de sus prácticas cotidianas, que se pueden definir como aquellas regularidades y rutinas que permiten la reproducción de las instituciones sociales (Novelo \& López, 2000) y los significados que los actores les confieren (Sharma \& Gupta, 2006: pp. 11-13).

\section{Muestra}

Este estudio se realizó en el estado de Veracruz, México. La selección de esta entidad responde a su importancia demográfica, pues se trata del tercer estado más poblado de México con más de siete millones de habitantes, y el que tiene mayor número de escuelas y docentes de la república (INEGI, 2011). También se consideró su desempeño educativo, que está por debajo de la media nacional 
(INEE, 2012), y su condición de ser uno de los estados que no ha tenido procesos de alternancia política a nivel estatal. En ese sentido, el desarrollo político de Veracruz se caracteriza por la reproducción del corporativismo posrevolucionario y por un Estado con pocas capacidades (Olvera, Zavaleta \& Andrade, 2012).

En el caso de la aproximación cualitativa, se entrevistó y se realizaron dos grupos focales a una muestra intencional de maestros que cumplieran con los criterios de estar trabajando frente a grupo en escuelas públicas de nivel básico (preescolar, primaria y secundaria) y que trabajaran en los municipios de Xalapa, Coatepec, Banderilla y San Andrés Tlalnehuayocan, los principales municipios que conforman la zona metropolitana de Xalapa.

Respecto de la aproximación cuantitativa se elaboraron dos bases de datos, una con peticiones y solicitudes dirigidas a la Coordinación de Delegaciones de la Secretaría de Educación de Veracruz (sev) que consideró 2783 demandas ciudadanas de 175 municipios del estado recibidas entre 2005 y 2009; y otra con notas de prensa estatal relacionadas con el tema educativo provenientes de 47 medios diferentes —impresos y electrónicos- que incluyó 1326 notas de prensa, y 234 notas relativas a protestas y manifestaciones. Se creó y aplicó un cuestionario a una muestra intencionada de 127 participantes de Consejos Escolares de Participación Social (CEPS) de 16 escuelas primarias de turno matutino, ubicadas en la zona metropolitana de Xalapa, de los que el $73.2 \%$ fueron mujeres, el $26.8 \%$ hombres, el $51.4 \%$ madres/padres de familia, y el $48.6 \%$ maestras/os y/o directoras/es.

\section{Instrumentos}

Lo instrumentos de recolección de la información fueron de dos tipos: para la etapa cualitativa se desarrolló un guion de entrevista/grupo focal centrado en las experiencias de participación, protestas y peticiones de los actores entrevistados, así como sus valoraciones sobre la legitimidad y utilidad de estos mecanismos. Para conocer el desempeño de los ceps se adaptó el "Cuestionario de desempeño de las instancias públicas de deliberación” (Hevia, Vergara-Lope \& Ávila, 2009), que desarrolla las escalas de institucionalidad, representatividad, y calidad e impacto de las actividades, y consta de cuarenta reactivos.

\section{Procedimientos}

Los procedimientos analíticos para los grupos focales se centraron en el estudio estructural del discurso (Martinic, 2006), mientras que los procedimientos para 
el análisis de bases de datos y cuestionarios fueron la estadística descriptiva no paramétrica, utilizando sobre todo frecuencias y tablas de contingencia.

\section{Resultados}

El primer resultado de esta investigación es que las relaciones cotidianas de interacción entre gobernantes y gobernados en el campo de la educación son heterogéneas, sin embargo, pueden agruparse en tres grandes dinámicas de participación: reglamentada, particularistas, y contenciosas.

\section{A. Dinámicas de participación reglamentada.}

Una primera dinámica de relación se establece de manera legal-normativa, por medio de la Ley General de Educación (LGE) y los lineamientos normativos de la Secretaría de Educación Pública (SEP). Estas dinámicas contienen la gestión de los órganos institucionales de participación social: las Asociaciones de Padres de Familia y los Consejos Escolares de Participación Social (CEPs).

Durante décadas el principal mecanismo de participación de los padres y madres de familia en la comunidad escolar fueron las Asociaciones de Padres de Familia. Estas, que no han modificado su normatividad desde 1980, cumplen funciones específicas, limitando su accionar en especial a la cooperación económica para el mejoramiento físico de las escuelas, y en responsabilizarse de cobrar y administrar las cuotas escolares (Martiniello, 1999). Históricamente estas asociaciones han estado impedidas de realizar acciones de gestión pedagógica en las escuelas, a pesar de cumplir con funciones de representación de los padres ante las autoridades educativas (Latapí, 2004).

Con la promulgación de la LGE en 1993, a las Asociaciones de Padres de Familia se sumó la creación de los Ceps. De acuerdo con esta ley, los CEPs permiten la participación de la comunidad y de los padres de familia en la vida escolar (Estados Unidos Mexicanos, 1993; sEP, 2010). Como reseña la literatura, a pesar de su inclusión por ley desde 1993, no fue hasta 2000 cuando se emitieron algunos criterios normativos para hacerlos funcionar, con la emisión de los Acuerdos Secretariales 260 para el Consejo Nacional de Participación Social, y 280 que establece los lineamientos generales de los Consejos de Participación Social (sEP, 1999, 2000). A pesar de este impulso legal, fue gracias al Programa Escuelas de Calidad donde estos consejos se instalaron de manera más sistemática (Martínez et al., 2010: p. 161 y ss.; Santizo, 2011).

Los resultados que se presentan a continuación corresponden a una encuesta que se llevó a cabo en el primer año de implementación de estos lineamientos (2011) en una zona metropolitana de Veracruz y coinciden con otras inves- 
tigaciones: los CEPS, aún bajo los lineamientos de 2010, resultan ser espacios institucionalizados pero poco útiles para resolver las múltiples necesidades de las escuelas.

De acuerdo con los lineamientos de 2010, los CEPs poseen al menos 16 funciones sustantivas establecidas, a las que se suman otras de carácter administrativo. Al preguntar a 127 participantes de estos consejos sobre el grado de cumplimiento de estas funciones en la zona metropolitana de Xalapa, el promedio de respuestas de las funciones fue de $8.48(\mathrm{DE}=4.8)$, o sea, que según los entrevistados cumplen con la mitad de sus funciones, ya que dijeron participar más en eventos artísticos y culturales, que en informes sobre los diversos recursos disponibles y evaluaciones escolares (tabla I).

Tabla I. Funciones de los CEPS según los entrevistados

\begin{tabular}{lllll}
\hline El CEPS de su escuela cumple con estas funciones... & Si & No & $\%$ Si & \% No \\
\hline Elaborar informe de transparencia sobre recursos federales, estatales, municipales & 35 & 92 & 27.6 & 72.4 \\
Evaluaciones escolares & 36 & 91 & 28.3 & 71.7 \\
Saber el monto de recursos otorgados a la escuela & 57 & 70 & 44.9 & 55.1 \\
Establecimiento de metas y acciones para mejorar el rendimiento escolar & 57 & 70 & 44.9 & 55.1 \\
Colabora en el mejoramiento infraestructura escolar & 58 & 69 & 45.7 & 54.3 \\
Eventos deportivos & 62 & 65 & 48.8 & 51.2 \\
Expone en asambleas los montos recibidos por la escuela & 63 & 64 & 49.6 & 50.4 \\
Supervisión de alimentos y bebidas & 63 & 64 & 49.6 & 50.4 \\
Promoción y mantenimiento del uso de la biblioteca & 63 & 64 & 49.6 & 50.4 \\
Elabora un informe de transparencia & 65 & 62 & 51.2 & 48.8 \\
Eventos para recaudar fondos & 69 & 58 & 54.3 & 45.7 \\
Colabora en la RdC de la APF & 71 & 56 & 55.9 & 44.1 \\
Elabora un informe de transparencia sobre APF & 71 & 56 & 55.9 & 44.1 \\
Expone en asambleas los informes & 77 & 50 & 60.6 & 39.4 \\
Elabora informe de resultados de las acciones que se desarrollarán & 81 & 46 & 63.8 & 36.2 \\
Eventos artístico culturales & 87 & 40 & 68.5 & 31.5 \\
\hline
\end{tabular}

Fuente: Hevia (2014: p. 242).

Al preguntar sobre la valoración de los CEPs y si cumplían con sus objetivos, el $47.3 \%$ afirmó que los objetivos de los consejos se cumplían "poco" o "nada" y un $20.7 \%$ consideró que los CEPs contribuyen "mucho" a mejorar la participación social.

Estos resultados identifican problemas de diseño y desempeño de los CEPS para cumplir con sus funciones. Por un lado, se advierte la falta de autonomía (el 70\% los presidentes de los consejos analizados son al mismo tiempo los directores) y escasos recursos de poder que les permitan transformarse en un espacio legítimo de reivindicación de demandas. Por otro lado, estos consejos se 
desarrollan en un contexto doblemente reacio a la participación, tanto desde las autoridades gubernamentales como desde los líderes sindicales que mantienen una simbiosis atípica que excluye de manera sistemática a los ciudadanos de las esferas de decisión en la educación (Latapí, 2004; Ornelas, 2008).

A estos problemas debe sumarse algunos déficit de legitimidad, no solo porque están creados "por decreto" desde arriba, sino porque duplican varias de las funciones con las Asociaciones de Padres de Familia, y algunos déficit de efectividad, puesto que, a pesar de que la normatividad permite que tengan funciones de vigilancia y monitoreo, en la práctica siguen acotándose a la participación como cooperación y mejoramiento escolar, con muy poco espacio para intervenir en otras esferas educativas.

\section{B. Dinámicas particularistas.}

Los déficit de participación vía consejos y asociaciones no significa, en todo caso, que las relaciones sociedad-Estado sean escasas en el campo de la educación. El análisis sistemático de los sistemas de atención ciudadana y de las acciones cotidianas de relación entre las autoridades y los ciudadanos permite ver la emergencia de una relación que trasciende el campo educativo, pero que se reproduce en su interior: las dinámicas particularistas.

La característica central de estas dinámicas es el predominio de factores personalistas para resolver un problema, apelando a una relación personal, más que institucional y basadas en el favor (no en el derecho). Estas dinámicas incluyen un amplio rango de sistemas de atención ciudadana, audiencias particulares, recepción de solicitudes por parte de las autoridades en sus giras de trabajo, sistemas individualizados de atención, peticiones diversas a la autoridad, etcétera, donde los ciudadanos buscan solucionar una demanda o problema específico por medio de su exposición por escrito a la autoridad para resolver de manera particular su situación, vinculándose de esta manera con el ejercicio al derecho de petición, a saber: el derecho a ser escuchado por la autoridad y el derecho a obtener una respuesta (Cienfuegos, 2004: p. 22).

Los puntos fundamentales del derecho de petición tienen que ver con la escucha y la respuesta por parte de la autoridad. Por ello los gobiernos se esfuerzan en generar un "ambiente de escucha" adecuado. Los sistemas de atención ciudadana se utilizan en giras donde la autoridad está presente, o en programas de acercamiento gubernamental, como los programas "Lunes Puertas Abiertas" o "Jornadas de Gobierno Itinerante" que se desarrollaron en Veracruz entre 2005 y 2010, bajo la administración de Fidel Herrera Beltrán.

El programa "Lunes Puertas Abiertas" buscó encarnar una forma innovadora y más cercana de gobernar, aunque tiene múltiples antecedentes reseñados en la literatura, especialmente en los "miércoles ciudadanos" que implementaron 
algunos gobiernos locales del PAN durante las décadas de 1990 y 2000 (Poot, 2000). Este programa consistía en que los responsables de todas las dependencias estatales atendieran directamente a la población. Así, cada lunes, entre las diez y las quince horas en todas las oficinas estatales de gobierno cada una de las direcciones y áreas tenían un stand en los espacios comunes de las secretarías o dependencias, atendiendo de manera directa a los ciudadanos que se acercaban a presentar sus inquietudes. Las "Jornadas de Gobierno Itinerante", por otro lado, consistían en concentrar la oferta de gobierno municipal en un municipio determinado para atender directamente las solicitudes y demandas de la población (Gobierno del Estado de Veracruz, 2007). En ambos casos, la presencia del responsable que está ahí para escuchar le dan sentido y legitimidad a estos mecanismos.

Por estas vías, el gobierno recibió miles de consultas cada año. Para esta investigación, se construyó una base de datos con demandas recibidas por la Coordinación de Delegaciones, la oficina encargada de la SEv de implementar ambos programas, así como atender el seguimiento de la demanda recibida en giras del gobernador y el secretario de Educación. Esta base permite caracterizar tanto el tipo de demanda como los actores sociales que utilizan esta vía para relacionarse con el gobierno. La tabla II muestra los principales actores que presentaron peticiones ante la autoridad.

Tabla II. Clasificación de actores que presentan solicitudes a la SEV

\begin{tabular}{lcc}
\hline & Frecuencia & Porcentaje \\
\hline Director/a escuela & 1101 & 39.6 \\
Ciudadanos particulares & 771 & 27.7 \\
Personal docente & 290 & 10.4 \\
Autoridades municipales & 205 & 7.4 \\
Sociedad de Padres de Familia & 179 & 6.4 \\
Organización social & 88 & 3.2 \\
Estudiantes & 22 & 0.8 \\
Otros & 127 & 4.6 \\
Total & 2783 & 100.0 \\
\hline
\end{tabular}

Fuente: Elaboración propia con base en Hevia (2014: p. 163).

Como puede verse, la gran mayoría de las solicitudes fueron presentadas por miembros de la comunidad escolar, principalmente por docentes (directores y maestros). Puesto que la mayoría de las solicitudes $(1711,61.5 \%)$ se recibieron por medio del programa Jornadas de Gobierno Itinerante, es plausible imaginar que los directores de las escuelas de los municipios donde se realizaron estas actividades aprovecharon la visita de las autoridades para registrar diversas solicitudes. 
Respecto al contenido de las demandas, de los 2783 casos solo en 1691 se puede identificar la autoridad a la que se dirige la petición: $69.5 \%$ de las demandas $(\mathrm{N}=1,175)$ estaban dirigidas al gobernador del Estado, y $14.5 \%(\mathrm{~N}$ = 246) dirigidas al secretario de educación. Esto refleja la importancia de los representantes del poder ejecutivo en los sistemas de atención ciudadana. Al analizar el objeto de las solicitudes, más de la mitad se relacionaban con infraestructura y mobiliario escolar, y enseguida se encontraban las solicitudes de trabajo y de apoyos económicos (tabla III).

Tabla III. Categoría de tipos de solicitudes recibidas en la SEV

\begin{tabular}{lcc}
\hline & Frecuencia & Porcentaje \\
\hline Infraestructura y mobiliario & 1441 & 51.8 \\
Solicitud trabajo y apoyos & 830 & 29.8 \\
Situaciones administrativas & 241 & 8.7 \\
Calidad. Cobertura educativa & 157 & 5.6 \\
Demás demandas & 114 & 4.1 \\
Total & 2783 & 100.0 \\
\hline
\end{tabular}

Fuente: Hevia (2014: p. 170).

Como resulta evidente, no se pueden desligar los problemas de cobertura, equidad y calidad de la situación socioeconómica del estado. Tal como señalan Backhoff et al:

el nivel socioeconómico de los estudiantes es la variable que de manera consistente se encuentra más asociada a diferentes indicadores de logro educativo [...] resulta fundamental para el logro educativo en países en vías de desarrollo la importancia de recursos materiales y humanos, como la infraestructura escolar, el tamańo de los grupos, la experiencia y la formación docente, y la disponibilidad de material didáctico (Backhoff et al., 2007: pp. 16-17).

Según el Consejo Nacional de Evaluación de la Política de Desarrollo Social (Coneval), el $58.5 \%$ de la población de Veracruz se encontraba en situación de pobreza en 2010 (4 466000 personas), de las cuales, el 39.2\% (2 995 000) eran consideradas pobres moderados y el 19.3\% (1 473 000) en situación de pobreza extrema. Además, un 24.1\% de la población (1 842000 personas) se consideraban pobres por poseer alguna carencia social, y 115000 habitantes, esto es, apenas el $13.3 \%$ de la población podían considerarse ni pobres ni vulnerables (Coneval, 2012: p. 11).

La pobreza y vulnerabilidad en la que viven la mayoría de los veracruzanos viene acompañada de altos índices de desigualdad. Según el Coneval, Veracruz 
es una de las cinco entidades con mayor grado de desigualdad del país medida con el índice de Gini, que en la entidad alcanzó en el año 2000 un valor de 0.5584 (sobre la media nacional que fue de 0.5458 ), y en 2005 un valor de 0.5136 (sobre la media nacional de 0.5006) (Coneval, 2011).

Estas carencias tienen un impacto en la infraestructura escolar y en la carencia de recursos básicos. Según el gobierno del estado, para el ciclo 20092010 la educación básica reportaba un rezago de infraestructura en 11410 planteles educativos, de los cuales 7468 necesitaban de construcción de aulas, laboratorios, talleres y anexos, y en 3942 planteles se requería de habilitación (Secretaría de Educación de Veracruz, 2011: p. 34 y ss.). Es decir, el 54\% del total de escuelas del estado. De igual manera, los niveles de educación media y superior presentaban un rezago en la construcción de 407 espacios educativos y 128 con necesidades de rehabilitación. Y se requería, al cierre de ese ciclo escolar, la sustitución de 811 planteles que se encontraban trabajando en aulas de madera, casas particulares y aulas temporales (Secretaría de Educación de Veracruz, 2011).

En términos analíticos, las consecuencias intencionales de la recepción de peticiones individuales para el gobierno son evidentes, puesto que adquieren legitimidad y difunden una imagen positiva, pero también hay consecuencias positivas para los ciudadanos, quienes ejercen su derecho a ser escuchados y a plantear su petición en forma directa al responsable.

Sin embargo, estos dispositivos generan consecuencias no intencionales que refuerzan y reproducen las asimetrías de poder entre ciudadanos y la autoridad, por medio del refuerzo de las dinámicas personalistas y particularistas de relación. Que el responsable esté ahí, y que por eso funcione este espacio, quiere decir que su presencia es determinante para que un trámite se pueda realizar. Esto hace que las decisiones sean percibidas como decisiones particulares del responsable en cuestión. Acceder a mobiliario escolar o conseguir trabajo terminan siendo, bajo esta lógica, decisiones voluntarias de las personas que están a cargo y no procesos estandarizados y decisiones razonadas. $Y$ esto lo saben tanto los ciudadanos como las autoridades. Los primeros usan de mejor manera posible sus relaciones personales, para que el responsable los considere; mientras que los segundos fortalecen esa idea, o al menos no la desmienten. Esto abre toda una gama de espacios de discrecionalidad que pueden terminar en actos de negligencia, corrupción y abusos de autoridad.

Con esta dinámica de relación, en la que se privilegia al responsable y su capacidad de decisión sobre las estructuras institucionales, se desincentiva la creación de estándares de atención, cada caso se toma como "particular" y se vuelve muy difícil para la misma institución generar normas comunes de respuesta. Estos sistemas son casuísticos, y no estandarizan su respuesta. Por ejem- 
plo, para el mobiliario escolar existía un trámite administrativo para que los directores o maestros realizaran su solicitud, y las oficinas de Oficialía Mayor, las encargadas de dar respuesta, tenían criterios establecidos para dotar de material a las escuelas, o en su defecto, para seleccionar a qué escuela se entregaría el mobiliario en caso de escasez presupuestal. Pero la existencia de peticiones, así como su relativo éxito al menos para hacerse escuchar, prevalecía sobre las rutinas administrativas. Por ello, maestros y directores comprometidos con sus escuelas refirieron utilizar diversos procedimientos para acceder a sus demandas, debilitando así el sistema estandarizado. Las excepciones terminan siendo más numerosas que la regla.

\section{Dinámicas contenciosas-disruptivas.}

Una tercera relación se puede denominar "dinámicas contenciosas-disruptivas" que, como su nombre lo indica, remiten a relaciones marcadas por el conflicto. Según el diccionario, "contencioso" se puede definir como conflicto, y/o como dicho de una persona "que por costumbre disputa o contradice todo lo que otras afirman" (Real Academia Española, 2011), de ahí que se use, por ejemplo, para designar tribunales de lo contencioso-administrativo. Sin embargo, en antropología política, su uso se asocia más tanto a las discusiones en torno a la "construcción cotidiana del Estado" (Roseberry, 2002), como al desarrollo de las investigaciones encabezadas por Tarrow, Tilly y MacAdam en torno a las dinámicas de contención. Según estos autores, las políticas contenciosas se pueden definir como una " [...] interacción pública, episódica y colectiva entre los hacedores de reclamos (makers of claims) y sus objetos cuando (a) al menos un gobierno es un demandante, un objeto de reclamaciones, o una parte de los reclamos y (b) las reivindicaciones, de realizarse, pueden afectar a los intereses de al menos uno de los reclamantes (McAdam, Tarrow \& Tilly, 2001: p. 5).

En este sentido, las relaciones contenciosas estarán caracterizadas por las disputas y acciones directas de grupos, colectivos, organizaciones y movimientos para lograr y exigir una respuesta gubernamental. Estas relaciones incluyen diversos repertorios de acción y presión, entre los que sobresale la protesta en las vías públicas (López, López-Saavedra, Tamayo \& Torres, 2010). Múltiples marchas y manifestaciones comienzan como resultado de la frustración de no haberse resuelto la demanda manifestada por medio del patrón de relaciones particularistas y se van transformando como acciones más efectivas para lograr tener la atención de la autoridad.

Bajo esta óptica, aquí se optó por analizar notas de prensa de entre enero de 2007 y diciembre de 2010 provenientes de 44 medios impresos y electrónicos del estado, incluyendo Diario de Xalapa, Imagen de Veracruz, El Dictamen y Milenio Portal, que concentraron más del 50\% de las 1326 notas de prensa 
relacionadas con el campo educativo en el periodo analizado. De esas notas, 234 correspondieron a diversos repertorios de manifestación, abarcando inconformidades públicas, manifestaciones en la vía pública, paros laborales y toma de oficinas/planteles, hasta llegar al boqueo de vialidades y lo que denominamos "autoviolencia", para definir a los repertorios que, para lograr el impacto deseado, atentan contra la propia seguridad de los manifestantes, tal como las huelgas de hambre o, como en el caso de la educación en Veracruz, amenazas con crucificarse por parte de un maestro como forma extrema para hacerse escuchar.

La tabla IV muestra los repertorios de manifestación según los actores sociales que utilizan dichos mecanismos. Como podrá verse, lo más reseñado por la prensa local son las acciones de manifestación de los sindicatos, a los que siguen actores pertenecientes a la comunidad escolar: padres de familia y maestros que hablan "en representación de" escuelas específicas.

Tabla IV. Repertorio de manifestación por tipo de actor en la prensa local, 2007-2010.

\begin{tabular}{lcccccc}
\hline Repertorio de manifestación & Sindicatos & Padres de familia & Estudiantes & Maestros & Otros & Total \\
\hline Inconformidad & 28 & 24 & 3 & 20 & 4 & 79 \\
$(\%)$ & 35.4 & 30.4 & 3.8 & 25.3 & 5.1 & 100.0 \\
Manifestación vía pública & 17 & 17 & 6 & 23 & 3 & 66 \\
$(\%)$ & 25.8 & 25.8 & 9.1 & 34.8 & 4.5 & 100.0 \\
Paro laboral & 13 & 1 & 0 & 6 & 1 & 21 \\
$(\%)$ & 61.9 & 4.8 & 0.0 & 28.6 & 4.8 & 100.0 \\
Toma oficina/planteles & 12 & 19 & 3 & 6 & 4 & 44 \\
$(\%)$ & 27.3 & 43.2 & 6.8 & 13.6 & 9.1 & 100.0 \\
Bloqueo vialidades & 8 & 5 & 2 & 6 & 0 & 21 \\
$(\%)$ & 38.1 & 23.8 & 9.5 & 28.6 & 0.0 & 100.0 \\
Autoviolencia & 0 & 0 & 0 & 3 & 0 & 3 \\
(\%) & 0.0 & 0.0 & 0.0 & 100.0 & 0.0 & 100.0 \\
Total & 78 & 67 & 14 & 63 & 12 & 234 \\
$(\%)$ & 100.0 & 100.0 & 100.0 & 100.0 & 100.0 & 100.0 \\
\hline Fule & & & & & &
\end{tabular}

Fuente: Elaboración propia.

Aquí se puede ver que mientras el sindicato usa de manera más frecuente las inconformidades, las manifestaciones en la vía pública y, como es obvio, el paro laboral, los padres de familia tienen que exponerse más por medio de la toma de oficinas y planteles y por bloqueo de vialidades, lo mismo que los estudiantes, los cuales se concentran en estos repertorios. Los maestros, por su parte, usan más la autoviolencia, el bloqueo de vialidades y las tomas de oficinas. Así, en el campo educativo en Veracruz, existe una serie amplia de repertorios de manifestación que buscan hacerse escuchar ante las autoridades 
y que pueden comprenderse desde un punto de vista incremental: van "aumentando" el grado de la violencia, comenzando desde la manifestación pública de inconformidad y la toma pacífica de la calle frente a la escuela, hasta concluir en casos extremos de violencia autoinflingida en la plaza central de la ciudad capital.

Como parece obvio al analizar casos específicos, la necesidad de "aumentar el volumen" es clave para hacerse escuchar, y las manifestaciones y protestas implican una enorme cantidad de energía únicamente para eso: para hacerse escuchar. Esto se relaciona de manera directa con el uso de los espacios vinculados a la violencia incremental. Las tendencias analizadas plantean el uso diferenciado de al menos tres espacios que parecen centrales para expresarse en la arena pública y así manifestarse: la escuela, las calles y la plaza pública.

Así, repertorios de manifestación y la violencia incremental se funden con estos espacios: en la plaza pública conviven las manifestaciones pacíficas con los actos más extremos de autoviolencia, y es este espacio el más utilizado por maestros y comunidad escolar para amplificar su voz. De la misma manera, las calles (tanto para manifestarse como para bloquearlas como acción de protesta) permiten la convivencia de repertorios más o menos incrementales, pero su uso es más frecuente por parte de maestros y sus sindicatos, por lo general actores más organizados, acostumbrados a utilizar esta clase de manifestación en marchas y festivales (como los del 1 de mayo o incluso como los del día en que se celebra la Independencia, cuando marchan las escuelas de todo el municipio por las calles), y conocedores de los símbolos y las relaciones "sintagmáticas" de las marchas (Rodríguez, 2010). Por último, la escuela como espacio en disputa que, por medio de las tomas, los actores de la sociedad parecen quererse reapropiar. Es necesario recordar que, a lo largo de la historia reciente del país, la construcción de las escuelas corrió a cargo, tanto en aportaciones económicas como en mano de obra, de la propia comunidad (Rockwell, 1995). Por eso tomar la escuela es un recurso recurrente de los padres de familia para aumentar su voz.

Para los maestros, y en cierta medida para los sindicatos, la escuela es el espacio de trabajo, el lugar laboral, donde de hecho se enfrentan a los padres de familia en la mayoría de las ocasiones para terminar en tomas de planteles. Quizá por esto prefieren las calles para reivindicar sus demandas como acto de presión, de "mostrar músculo", mientras están presentes en las mesas de negociación con las autoridades. Los padres de familia, al tomar los planteles, aspiran a eso: a ejercer el derecho a ser escuchados y poder negociar sus demandas con la autoridad, y como no tienen espacios predeterminados para ello, tienen que arriesgar más, ya sea su seguridad jurídica (Hevia, 2011) ya su integridad física. 


\section{Conclusiones}

Las conclusiones pueden ordenarse en torno a dos discusiones, una al interior del campo de la educación y otra más general en relación con las dinámicas de participación.

En términos más específicos al interior del campo educativo, la identificación de estos patrones de relación, y su coexistencia, representan desafíos mayúsculos para la gestión escolar, en especial el uso personalista de relación y la asimetría de poder que contiene.

Como se intentó mostrar, la comunicación entre autoridades y la comunidad escolar normativamente descansa en la creación de consejos de participación social. Sin embargo, los consejos escolares son solo una más de las rutas que los actores emplean para interactuar con las autoridades educativas. Sin embargo, la escasa participación de los ciudadanos en instancias reglamentadas no significa ausencia de relación. Como mostramos, los consejos coexisten con otros patrones, donde sobresalen las "relaciones particularistas". Estas incluyen un enorme rango de interacciones que se generan en las oficinas de atención ciudadana, audiencias particulares, recepción de solicitudes por parte de las autoridades en sus giras de trabajo, sistemas individualizados de atención, peticiones diversas a la autoridad, etcétera.

Esto permite comprender estas acciones en un contexto mayor: la atención ciudadana y la gestión se convierten en un mecanismo constante de relación que tienen las autoridades políticas con sus bases electorales y sus votantes. En estas relaciones, los políticos invierten mucho tiempo (Grindle, 2007) y promueven una forma de relación subordinada (Hevia, 2011), donde los demandantes piden ser "gobernados" (Chatterjee, 2008) y solicitan que los gobernantes se encarguen de resolver las demandas puntuales. Estas relaciones no se basan en una lógica de derechos ni de ciudadanía, sino que apelan al favor y la reciprocidad producto del trato directo, y por lo tanto, las demandas (y sus respuestas) son personalizadas, no estandarizadas. Estas relaciones permiten y premian la creación de intermediarios y de relaciones clientelares (Gómez Álvarez, 2009), reproducen el vínculo favor-agradecimiento como la base del contrato entre gobernantes y gobernados (Schedler, 2004) y fortalecen la idea del Estado como una "máquina generadora de esperanza" (Hope-Generating State Machine), donde todo es posible si se siguen los trámites y procedimientos formales (Nuijten, 2003: p. 16). A diferencia de solicitudes administrativas o quejas presentadas por sujetos de derecho, las relaciones particularistas apelan, por un lado, a reconocer y reproducir la diferencia de poder entre gobernantes y gobernados, y por otro, a personalizar la relación con miras a que el gobernado reciba los favores del gobernante y pueda así tener una respuesta positiva. 
En este sentido, las relaciones particularistas pueden resultar más efectivas que otras formas de relación y resolver problemas específicos no contemplados en la norma, así como situaciones extraordinarias, pero disminuyen las capacidades técnico-administrativas del Estado debido a que dificultan la estandarización de los procesos y la toma de decisiones basadas en el cálculo político más que en la planeación.

En este sentido, la hegemonía de este patrón de relación impacta negativamente sobre la capacidad estatal de respuesta, puesto que prioriza la respuesta individual y políticamente orientada en vez del establecimiento de estándares de atención más igualitarios, lo que generaría respuestas diferenciadas según la fuerza del interlocutor. Por eso la importancia que adquieren las relaciones contenciosas.

Las denominadas "relaciones contenciosas-disruptivas" implican todas las disputas y acciones directas de personas, grupos, colectivos, organizaciones y movimientos para lograr y exigir una respuesta gubernamental. Estas relaciones incluyen diversos repertorios de acción y presión, entre las que sobresale la protesta en las vías públicas (López et al., 2010). Múltiples marchas y manifestaciones comienzan por la incapacidad estatal para resolver las demandas planteadas y como resultado de la frustración ante la inutilidad de los sistemas institucionales de comunicación entre gobernantes y gobernados, entre otras causas. Las acciones disruptivas se transforman en las acciones más efectivas para lograr tener la atención de la autoridad y exigir respuesta a las autoridades estatales. Así, en todo el país se reproducen manifestaciones, tomas de palacios municipales y oficinas para solucionar cientos de demandas particulares: cumplir con una obra, expulsar a un maestro faltista, solucionar un deslinde de tierras, liberar a personas injustamente presas, etcétera (Martí i Puig, 2010).

En términos de efectividad política, las consecuencias de las relaciones contenciosas-disruptivas son mixtas. Por un lado, se generan grados crecientes de institucionalización informal de la atención a la demanda por esta vía (por lo general los gobiernos reportan en sus informes oficiales como "resultados" la atención de grupos de protesta, existe un protocolo bien establecido para atender y desactivar estas marchas en los palacios de gobierno estatales y municipales), y reproducen liderazgos de tipo carismático y relaciones corporativas entre los líderes de la organización/movimiento, operadores políticos-partidarios y las amplias bases sociales, usando el acarreo y otras medidas como formas de demostración de fuerza política (Hernández, 1992). Pero por otro constituye prácticamente el único instrumento que tienen los pobres y los colectivos invisibilizados para acceder a la voz en el espacio público (Tamayo, 2010). Con instancias participativas y representativas que poseen serios problemas de sobrerrepresentación de los poderosos y subrepresentación de los oprimidos (Cunill, 
1997), las movilizaciones y protestas sociales han sido actores fundamentales en la región para expandir derechos, así como para redefinir las relaciones de poder entre gobernantes y gobernados (Eckstein, 2001). De ahí que se pueda observar un uso creciente de protestas ante la incapacidad de los gobiernos para resolver demandas ciudadanas.

En relación con la discusión más general, cabe discutir si la propuesta de identificar a la participación ciudadana como un patrón de relación sociedadEstado permite valorar la capacidad de estos dispositivos para cumplir con las expectativas que de ellos se tiene. En efecto, una discusión constante en los análisis sobre estos dispositivos tiene que ver con sus efectos en diversas áreas: innovación democrática, capacidad de incidencia o ampliación del espacio público, generación de políticas redistributivas, etc. Es en estos procesos donde diversos analistas advierten del riesgo que implica sobrecargar de expectativas tanto a los propios dispositivos participativos (Font, 2015) como a las capacidades de los ciudadanos y sus organizaciones para incidir en lo público por medio de estos dispositivos (Leiras, 2007). De esta forma, al definir las relaciones de participación reglamentada como una forma, de hecho no protagónica, de relación sociedadgobierno, que coexiste con otras dinámicas de mayor estabilidad y legitimidad social en el tiempo, permite ajustar las expectativas sobre las potencialidades de esos dispositivos como instrumentos de cambio o transformación social y situarlos en una dimensión menos normativa. Como muestran los resultados, el derecho a incidir en el espacio público para el caso de la educación en Veracruz en el periodo analizado estuvo acotado y limitado según los términos que el propio gobierno dispuso, dando preferencia a la generación de relaciones particularistas, y por tanto, limitando a los ciudadanos a ser gobernados, en el sentido de Chatterjee (2008), y alejándose así de la retórica participacionista, y mostrando en la práctica los límites de esta retórica para el fortalecimiento democrático.

Para finalizar, queda una amplia agenda de investigación, tanto para el caso específico de la educación como para la discusión sobre participación institucional. En este sentido, creemos necesario, por un lado, profundizar el análisis en las diversas interacciones que estos patrones tienen entre sí, sobre todo a nivel de escuela. En efecto, es en la escala analítica local, estudiando escuelas específicas, donde es posible identificar los tipos de relación que existen entre las dinámicas descritas: ¿̨bajo qué contextos escolares, de gestión directiva, de trayectorias participativas de la sociedad en general conviven, o se complementan estas dinámicas?, ¿̨la institucionalización creciente de los consejos y el esfuerzo por dotarlos de contenido alterarán en el tiempo las dinámicas particularistas o disruptivas? Son preguntas que considero relevantes para una agenda más amplia. Por otro lado, resulta pertinente analizar bajo esta propuesta analítica los efectos de los recientes cambios administrativos en la política 
educativa mexicana, marcadamente con el fortalecimiento de la "autonomía de gestión escolar", expresados en lineamientos publicados en marzo de 2014 para fortalecer la autonomía en las escuelas, ¿se fortalecerán o modificarán estas dinámicas? De igual forma, es necesario identificar la recurrencia o no de estos patrones en otras áreas de política y otros territorios que permita conocer si lo que pasa en el campo de la educación es parte de la norma o de la excepción cuando se analizan las relaciones sociedad-Estado en México. Por último, se hace necesario discutir, dentro de la agenda más amplia del fortalecimiento democrático de la región, los efectos acumulativos de estas dinámicas en la construcción cotidiana del Estado: tanto desde la dimensión de políticas públicas para democratizar las relaciones gobernantes-gobernados (O’Donnell, 2007), como desde el lado de las prácticas cotidianas que van reproduciendo a las instituciones estatales, tal como plantean Sharma \& Gupta: "a veces en silencio, sin llamar la atención sobre sí mismas, y otras veces por medio de afirmar su presencia y poder, a través del trabajo diario de las burocracias" (Sharma $\&$ Gupta, 2006: p. 13).

\section{Referencias}

Aguilar Villanueva, L. F. (2011). Gobernanza y gestión pública. México: Fondo de Cultura Económica.

Alaníz Hernández, C. (2013). Modalidades de participación social en educación básica. Argumentos, 26(72), 167-189.

Alonso, G. V. (2007). Elementos para el análisis de capacidades estatales. En Alonso, G. V. (Ed.). Capacidades estatales, instituciones y politica social (pp. 17-40). Buenos Aires: Prometeo Libros Editorial.

Altman, D. (2005). Democracia directa en el continente americano: ‘autolegitimación gubernamental o censura ciudadana? Politica y Gobierno, XII(2), 203-232.

Anderson, J. A. (2005). Accountability in Education. Education Policy Series. París: IIEP, Unesco. Recuperado de http://www.iaoed.org/files/Edpol1.pdf

Auyero, J., Lapegna, P., \& Poma, F. P. (2009). Patronage Politics and Contentious Collective Action: A Recursive Relationship. Latin American Politics and Society, 51(3), 1-31.

Avritzer, L. (2010). Las instituciones participativas en el Brasil democrático (1a ed.). Xalapa, Veracruz: Universidad Veracruzana. 
Backhoff, E., Bouzas, A., Contreras, C., Hernández, E., \& García, M. (2007). Factores escolares y aprendizaje en México. El caso de la educación básica. México: InEe. Recuperado de http:// www.inee.edu.mx/images/stories/Publicaciones/Reportes_investigacion/Factores_escolares/Completo/factores_escolares_completo.pdf

Bauch, P. A. \& Goldring, E. B. (1998). Parent-Teacher Participation in the Context of School Governance. Peabody Journal of Education, 73(1), 15-35.

Bush, T. \& Glover, D. (2013). School Management Teams in South Africa: A Survey of School Leaders in the Mpumalanga Province. International Studies in Educational Administration (Commonwealth Council for Educational Administration y Management (CCEAM)), 41(1), 21-40.

Canales, A. (2006). La participación social en educación: un dilema por resolver. Perfiles Educativos, XXVIII(3), 62-80.

Carvalho, M. E. de. (2000). Rethinking Family-school Relations: A Critique of Parental involvement in Schooling. Taylor y Francis.

Castanheira, P. \& Costa, J. A. (2011). From a Collegially Elected Council to a Director: The Evolution of Portuguese School Management. Procedia-Social and Behavioral Sciences, (15), 2007-2011.

Chatterjee, P. (2008). La nación en tiempo heterogéneo y otros estudios subalternos. Buenos Aires: Clacso/Siglo Veintiuno Editores Argentina.

Cienfuegos, D. (2004). El derecho de petición en México. México: IIJ-unam.

Coneval. (2011). Mapas de Desigualdad 2000-2005. Recuperado el 12 de diciembre de http:// www.coneval.gob.mx/cmsconeval/rw/pages/medicion/mapas_de_desigualdad_2000_2005 /index.es.do

CONEVAl. (2012). Informe de pobreza y evaluación en el estado de Veracruz 2012. CONEVAL. Recuperado de http://web.coneval.gob.mx/coordinacion/entidades/Documents/Veracruz /principal/30informe2012.pdf

Cunill, N. (2012). ¿Qué ha pasado con lo público en los últimos 30 años? Balance y perspectivas. Revista del CLAD Reforma y Democracia, (52), 5-44.

Cunill, N. (1997). Repensando lo público a través de la sociedad. Caracas: CLAD.

Dagnino, E. (2006). Sociedad civil, participación y ciudadanía ¿¿De qué estamos hablando? En Isunza Vera, E. \& Olvera, A.(Eds.). Democratización, rendición de cuentas y sociedad civil: 
participación ciudadana y control social (pp. 223-242). México: MA Porrúa/Ciesas/Universidad Veracruzana.

Delgado-Gaitan, C. (1991). Involving Parents in the Schools: A Process of Empowerment. American Journal of Education, 100(1), 20-46.

Dietz, M. J. (Ed.). (2004). School, Family And Community: Techniques And Models For Successful Collaboration. Jones y Bartlett Learning.

Driessen, G., Smit, F. \& Sleegers, P. (2005). Parental Involvement and Educational Achievement. British Educational Research Journal, 31(4), 509-532.

Eckstein, S. (Ed.). (2001). Power and popular protest: Latin American Social Movements (Updated and expanded ed.). Berkeley: University of California Press.

Epstein, J. L. (2001). School, Family, and Community Partnerships: Preparing Educators, and Improving Schools. Westview Press.

Estados Unidos Mexicanos. (1993). Ley General de Educación. México: Dof 13 junio 1993. Últimas reformas: DOF 9 abril 2012.

Estrada, M. (2008). La participación social en la educación: hacia una "comunidad escolar" en Las Margaritas, Chiapas. Revista Interamericana de Educación para la democracia, 1(2), 185-204.

Estrada, M. (2014). La prospectiva de la participación social en la educación en México. El punto de vista de los especialistas. Revista Brasileira de Educação, 19(58), 731-753.

Font, J. (2015). ¿Para qué y por qué promover la participación ciudadana? Seminario internacional sobre políticas comparadas para la instrumentación de mecanismos de participación en América Latina. México: PNUD.

Gann, N. (1998). Improving School Governance: How Better Governors Make Better Schools. Washington, D. C.

Gleeson, D. \& Husbands, C. (2003). Modernizing Schooling Through Performance Management: A Critical Appraisal. Journal of Education Policy, 18(5), 499-511.

Gobierno del Estado de Veracruz. (2007). Segundo informe de gobierno. Lic. Fidel Herrera Beltrán. Xalapa: Gobierno del Estado de Veracruz.

Gómez Álvarez, D. (Ed.). (2009). Candados y contrapesos: la protección de los programas, politicas y derechos sociales en México y América Latina. Guadalajara: PNUD/ITESO. 
Grindle, M. S. (2007). Going Local: Decentralization, Democratization, and the Promise of Good Governance. Princeton: Princeton University Press.

Gurza Lavalle, A. \& Zaremberg, G. (2015). Más allá de la representación y del clientelismo: hacia un lenguaje de la intermediación política. Revista Mexicana de Ciencias Políticas y Sociales, 59(221). Recuperado el 19 de mayo de 2015 de http://www.revistas.unam.mx/index. $\mathrm{php} / \mathrm{rmspys} /$ article/view/47571

Henderson, A. T. \& Mapp, K. L. (Eds.). (2002). A New Wave of Evidence. The Impact of School, Family, and Community Connections on Student Achievement. Austin: SEDL.

Hernández, R. (1992). ¿¿Del corporativismo a la contienda electoral? En Luna M. \& Pozas Horcasitas, R. (Eds.). Relaciones corporativas en un periodo de transición (pp. 149-171). México: IIS-UNAM.

Hevia, F. J. (2014). Peticiones, protestas y participación. Patrones de relación sociedad-gobierno en la educación básica en Veracruz a inicios del siglo XXI. México: CIESAS.

Hevia, F. J. (2011). Poder y ciudadanía en el combate a la pobreza: el caso de Progresa/Oportunidades de México. Bruselas: Peter Lang Pub Inc.

Hevia, F. J. (2010). Participación ciudadana institucionalizada: análisis de los marcos legales de la participación en América Latina. En Dagnino, E., Olvera, A. \& Panfichi A. (Eds.), La disputa por la construcción democrática en América Latina (2a. ed.) (pp. 367-395). México: Fondo de Cultura Económica/Universidad Veracruzana/ciesas.

Hevia, F. J. (2009). Relaciones sociedad-Estado. Análisis interactivo para una antropología del Estado. Espiral, XV(45), 43-70.Hevia, F. J., \& Vergara-Lope, S. (2011). ¿Cómo medir la participación? Creación, validación y aplicación del Cuestionario Conductas de Participación. México: CIESAS/Indesol.

Hevia, F. J., Vergara-Lope, S. \& Ávila, H. (2011). Participación ciudadana en México: consejos consultivos e instancias públicas de deliberación en el gobierno federal. Perfiles Latinoamericanos, 19(38), 65-88.

Hevia, F. J., Vergara-Lope, S. \& Ávila, H. (2009). Construcción de línea base para posteriores evaluaciones de impacto sobre la inclusión de las Organizaciones de la Sociedad Civil (OSC) en instancias públicas de deliberación. Informe final de investigación (Informe final de investigación). México: CIESAS/Indesol.

Hornby, G. \& Lafaele, R. (2011). Barriers to Parental Involvement in Education: An Explanatory Model. Educational Review, 63(1), 37-52. 
Huerta, E. (2010). Formas de participación parental en las escuelas secundarias mexicanas de altos y bajos resultados académicos. Revista Iberoamericana de Educación, (54), 167-185.

INEE. (2012). Banco de Indicadores Educativos. Recuperado el 6 de enero de 2012 de http:// www.inee.edu.mx/index.php/bases-de-datos/banco-de-indicadores-educativos

INEGI. (2011). Anuario estadístico de los Estados Unidos Mexicanos 2010. México: INEGI.

Isunza Vera, E. \& Gurza Lavalle, A. (Eds.). (2010). La innovación democrática en América Latina: tramas y nudos de la representación, la participación y el control social. México: CIESAS/ Universidad Veracruzana.

Latapí, P. (2004). La SEP por dentro: las politicas de la Secretaría de Educación Pública comentadas por cuatro de sus secretarios (1992-2004). México: Fondo de Cultura Económica.

Latapí, P. (1997). La participación social en educación. En Ezpeleta, J. (Ed.). Investigación educativa, gestión y participación social. Tomo 2 de investigación educativa. 1993-1995 (pp. 15-27). México: COMIE-DIE.Leiras, M. (2007). La incidencia de las organizaciones de la sociedad civil en las políticas públicas. Definiciones, explicaciones y evaluaciones de la literatura especializada local e internacional. En Acuña, C. \& Vacchieri, A. (Eds.). La incidencia política de la sociedad civil (pp. 17-65). Buenos Aires: Siglo Veintiuno Editores Argentina.

Lissidini, A., Welp, Y. \& Zovatto, D. (2008). Democracia directa en Latinoamérica. Buenos Aires: Prometeo Libros.

López Gallegos, A., López-Saavedra, N., Tamayo, S. \& Torres Jiménez, R. (Eds.). (2010). Yo no estuve ahi pero no olvido. La protesta en estudio. México: UAM.

López, P. (2010). Variables asociadas a la gestión escolar como factores de calidad educativa. Estudios Pedagógicos, XXXVI(1), 147-158.

Martí i Puig, S. (2010, octubre 6). Un análisis de la politica disruptiva en México ¿quiénes se movilizan y por qué lo hacen? Presentado en LASA-Meeting, Toronto.

Martin, C. J. (2010). Las innovaciones de la educación popular en el mundo jerárquico de las políticas públicas. En Barba. B. \& Zorrilla, M. (Eds.), Innovación social en educación: una base para la elaboración de politicas públicas (pp. 46-73). Aguascalientes: Universidad Autónoma de Aguascalientes/Siglo Veintiuno Editores.

Martínez, A., Bracho, T. \& Martínez, C. (2010). Los Consejos de Participación Social en la Educación y el Programa Escuelas de Calidad: ¿̨mecanismos sociales para la rendición de cuentas? En Olvera, A. (Ed.), La democratización frustrada. Limitaciones institucionales y 
colonización politica de las instituciones garantes de derechos y de participación ciudadana en México (pp. 129-174). México: CIESAs/Universidad Veracruzana.

Martinic, S. (2006). El estudio de las representaciones y el Análisis Estructural de Discurso. En Canales, M. (Ed.), Metodologias de investigación social: introducción a los oficios (pp. 299-320). Santiago: LOM.

Martiniello, M. (1999). Participación de los padres en la educación: Hacia una taxonomía para América Latina. Harvard Inst. for Internat. Development, Harvard Univ. Recuperado el 29 de abril de 2013, de http://pdf.usaid.gov/pdf_docs/PNACH684.pdf\#page=205McAdam, D., Tarrow, S. G. \& Tilly, C. (2001). Dynamics of Contention. Cambridge: Cambridge University Press.

Merino, M. (Ed.). (2010). ¿Qué tan público es el espacio público en México? México: FCE; Conaculta/Universidad Veracruzana.

Novelo, V. \& López Ramos, S. (Eds.). (2000). Etnografía de la vida cotidiana. México: MA Porrúa.

Nuijten, M. (2003). Power, community and the State: The Political Anthropology of Organisation in Mexico. Londres: Pluto Press.

Observatorio Ciudadano de la Educación. (2008). Participación social en educación: del análisis a las propuestas. México: Observatorio Ciudadano de la Educación.

O’Donnell, G. A. (2007). Hacia un Estado de y para la democracia. En Mariani, R. (Ed.). Democracia/Estado/Ciudadanía: hacia un Estado de y para la democracia en América Latina (pp. 25-62). Lima: PNUD.

Olvera, A., Zavaleta, A. \& Andrade, V. (Eds.). (2012). Los retos del desarrollo, la democracia, la sociedad civil y el medio ambiente en Veracruz. Xalapa: Covecyt-uv.

Ornelas, C. (2008). Politica, poder y pupitres: critica al nuevo federalismo educativo. México: Siglo XXI.

Paletta, A. (2012). Public Governance and School Performance. Public Management Review, 14(8), 1125-1151.

Parés, M. (Ed.). (2009). Participación y calidad democrática. Barcelona: Ariel.

PNUD. (2015). Seminario internacional sobre políticas comparadas para la instrumentación de mecanismos de participación en América Latina. México: PNUD. 
Poot, E. (2000). La renovada participación ciudadana: elecciones, organizaciones cívicas y nuevas formas de gobierno municipal. En Ramírez Saíz, J. M. (Ed.). Cambio político y participación en México: actores, movimientos y organizaciones (pp. 107-117). Guadalajara: СеРСом/UdG.

Real Academia Española. (2011). Diccionario de la lengua española. Vigésima segunda edición. Recuperado el 6 de abril de 2011, de http://buscon.rae.es/draeI/

Rockwell, E. (Ed.). (1995). La escuela cotidiana. México: Fondo de Cultura Económica.

Rodríguez Saldaña, E. (2010). Imágenes del actor colectivo. Una aproximación a la dinámica de las marchas de protesta en la Ciudad de México. Nueva Antropología, XXIII(72), 81-101.

Rojas, M. B. \& Ramírez, S. V. (2007). Gestión escolar y éxito académico en condiciones de pobreza. REICE. Revista Iberoamericana sobre Calidad, Eficacia y Cambio en Educación, 5(1), 121-144.

Roseberry, W. (2002). Hegemonía y lenguaje contencioso. En Joseph, G. M. \& Nugent, D. (Eds.), Aspectos cotidianos de la formación del Estado (pp. 213-226). México: Ediciones Era.

Santizo, C. (2011). Gobernanza y participación social en la escuela pública. Revista Mexicana de Investigación Educativa, 16(50), 751-773.

Santizo, C. (2006). Mejorando la rendición de cuentas y la transparencia a través de la participación social: el Programa Escuelas de Calidad en México. REICE, 4(1), 1-14.

Schedler, A. (2004). "El voto es nuestro". Cómo los ciudadanos mexicanos perciben el clientelismo electoral. Revista Mexicana de Sociología, 66(1), 57-97.

Secretaría de Educación de Veracruz. (2011). Programa Veracruzano de Educación 2011-2016. Xalapa: Secretaría de Educación de Veracruz.

Selee, A. D. \& Peruzzotti, E. (Eds.). (2009). Participatory Innovation and Representative Democracy in Latin America. Baltimore: The Johns Hopkins University Press.

SEP. (2010, 8 de junio). Acuerdo Número 535 por el que se establecen los lineamientos generales para lo operación de los Consejos Escolares de Participación Social. México: DoF.

SEP. (2000, 4 de agosto). Acuerdo Número 280 por el que se establecen los lineamientos generales a los que se ajustarán la constitución y funcionamiento de los Consejos de Participación Social en Educación. México: DOF.

SEP. (1999, 13 de agosto). Acuerdo Número 260 por el que se establecen los lineamientos para la constitución y funcionamiento del Consejo Nacional de Participación Social en la Educación. México: Dof. 
Sharma, A. \& Gupta, A. (2006). Introduction: Rethinking Theories of the State in an Age of Globalization. En Sharma, A. \& Gupta, A. (Eds.), The Anthropology of the State: A Reader (pp. 1-41). Malden, M. A.: Blackwell Pub.

Tamayo, S. (2010). Crítica de la ciudadanía. México: Siglo XXI Editores.

Welp, Y. \& Serduilt, U. (Eds.). (2009). Armas de doble filo: la participación ciudadana en la encrucijada. Buenos Aires: Prometeo Libros.

Zaremberg, G. (Ed.). (2012). Redes y jerarquias: representación, participación y gobernanza local en América Latina (vol. I). México: Flacso México.

Zurita, Ú. (2013). Paradojas y dilemas de la participación social en la educación básica en México. Apuntes, XL(72), 85-115.

Recibido el 19 de mayo de 2015. Aceptado el 30 de abril de 2016. 